\title{
The impact of gravity waves rising from convection in the lower atmosphere on the generation and nonlinear evolution of equatorial bubble
}

\author{
E. Alam Kherani ${ }^{1}$, M. A. Abdu ${ }^{1}$, E. R. de Paula ${ }^{1}$, D. C. Fritts ${ }^{2}$, J. H. A. Sobral ${ }^{1}$, and F. C. de Meneses Jr. ${ }^{1}$ \\ ${ }^{1}$ Instituto Nacional de Pesquisais Espaciais, Sao Jose dos Campos, SP, Brasil \\ ${ }^{2}$ NorthWest Research Associates, Colorado Research Associates Division, Boulder, USA
}

Received: 24 April 2008 - Revised: 20 March 2009 - Accepted: 20 March 2009 - Published: 7 April 2009

\begin{abstract}
The nonlinear evolution of equatorial F-region plasma bubbles under varying ambient ionospheric conditions and gravity wave seeding perturbations in the bottomside F-layer is studied. To do so, the gravity wave propagation from the convective source region in the lower atmosphere to the thermosphere is simulated using a model of gravity wave propagation in a compressible atmosphere. The wind perturbation associated with this gravity wave is taken as a seeding perturbation in the bottomside F-region to excite collisional-interchange instability. A nonlinear model of collisional-interchange instability (CII) is implemented to study the influences of gravity wave seeding on plasma bubble formation and development. Based on observations during the SpreadFEx campaign, two events are selected for detailed studies. Results of these simulations suggest that gravity waves can play a key role in plasma bubble seeding, but that they are also neither necessary nor certain to do so. Large gravity wave perturbations can result in deep plasma bubbles when ionospheric conditions are not conducive by themselves; conversely weaker gravity wave perturbations can trigger significant bubble events when ionospheric conditions are more favorable. But weak gravity wave perturbations in less favorable environments cannot, by themselves, lead to strong plasma bubble responses.
\end{abstract}

Keywords. Ionosphere (Ionospheric irregularities; Plasma waves and instabilities)

\section{Introduction}

The collisional interchange instability (CII) is believed to play a crucial role in the onset and development of equatorial spread F (ESF) turbulence (Haerendel, 1973). Radar

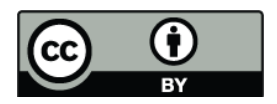

Correspondence to: E. Alam Kherani (alam@dae.inpe.br) observations of ESF reveal the existence of plumes that may penetrate to the topside F-layer and attain very high altitudes (Kelley et al., 1981). These plumes are identified as large-scale depletions or plasma bubbles and are believed to be generated by CII and Rayleigh-Taylor instability mechanism (Sultan, 1996). Numerous theoretical and numerical studies have been performed to assess the linear and nonlinear aspects of these complex dynamics (Scannepieco and Ossakow, 1976; Zalesak, 1979; Zargham and Seyler, 1987; Raghavarao et al., 1992; Huang et al., 1993; Keskinen et al., 2003). These studies have revealed the generation of rising bubbles initiated by a seed perturbation at bottomside of a rising F-layer. The seed perturbation used is either in the form of plasma density perturbation (Ossakow et al., 1979; Sekar et al., 1994; Kherani et al., 2005) or of wind perturbation, that subsequently cause density perturbation (Huang and Kelley, 1996).

The precise nature of the seed perturbations is one of the issues that has remained elusive and therefore constitutes one of the major focus of the Spread F Experiment (SpreadFEx) (Fritts et al., 2009, and reference therein). Rottger (1981) and Kelley et al. (1981) were the first to note the potential importance of gravity waves (GWs) as a seeding perturbation for the spread F. Thereafter, many researchers have experimentally suggested that GWs propagating in the equatorial thermosphere play an important role in seeding the bubbles and spread F (Kelley, 1986; Hysell et al., 1990). More evidence, based on in-situ measurements, as to the role of GW in the spread F has been provided by Singh et al. (1997) and McClure et al. (1998). Recent ground-based optical, radio and GPS observations at Kototabang, Indonesia also suggest the existence of a meaningful correlation between scintillations activity and gravity wave activity arising from the tropospheric convection (Ogawa et al., 2006). On theoretical side, Huang et al. (1993) and Huang and Kelley (1996) have studied the role of GWs in plasma bubble seeding using numerical simulation model of the CII mechanism. It was

Published by Copernicus Publications on behalf of the European Geosciences Union. 
Table 1. Important ionospheric, bubble and GW activity parameters from the observations.

\begin{tabular}{llll}
\hline Events & $\begin{array}{l}\text { Pre-reversal time (UT) } \\
\text { and peak velocity }\left(m s^{-1}\right)\end{array}$ & $\begin{array}{l}\text { Time of bubble occurrence (UT) } \\
\text { and altitude extension }(\mathrm{km})\end{array}$ & $\begin{array}{l}\text { Time of periodic modulation } \\
\text { and nature of downward phase propagation }\end{array}$ \\
\hline $\begin{array}{l}\text { Event 1: } \\
\text { 23-24 Oct }\end{array}$ & $21: 40,33$ & $22: 10,600$ & $19-20$, weak \\
24-25 Oct & $21: 00,18$ & $22: 15,450$ & $19-20$, strong \\
\hline Event 2: & (pre-reversal) (post-reversal) & & 19-20, weak \\
2-3 Oct & $(21: 20,18)(22: 30,12)$ & $22: 50,400$ & $19-21$, strong (almost 1.25 times stronger than 24-25 Oct) \\
5-6 Oct & $(21: 20,14)(23: 10,10)$ & $23: 30,400$ & \\
\hline
\end{tabular}

found that gravity waves with wind amplitude of a few meter per second, horizontal wavelength of few hundred kilometers and vertical wavelength of few tens of kilometers are a very effective seed mechanism for production of ESF. These studies clearly indicate the potential importance of GWs in the seeding process, but definitive measurements confirming the simultaneous occurrence of GWs and bubble seeding remained out of reach.

\section{Spread F observations and linear analysis of the CII mechanism}

The SpreadFEx campaign was conducted with a primary objective to quantify and understand the importance of GWs, arising from deep tropical convection, on spread F initiation at the bottomside F-layer over the Brazilian equatorial region. The campaign period was September to November 2005, during which spread F were observed on several nights (Fritts et al., 2009; Abdu et al., 2009). Spread F activity was found to vary day-to-day depending on the ambient ionospheric conditions and GW characteristics (Fritts et al., 2009; Abdu et al., 2009). In a companion paper by Abdu et al. (2009), the digisonde and VHF radar observations of spread F activity during SpreadFEx campaign are analyzed in detail. To study the likely importance of GW contributions to observed spread F activity, Abdu et al. (2009) have presented observations on few selected nights for which it was possible to estimate both mean ionospheric motions and GW contributions independently. The two set of events were chosen to examine the role of GW in the seeding of bubble and they are listed in Table 1.

The first event has two consecutive nights 23-24 October and 24-25 October 2005. On 23-24 October, the peak prereversal enhancement (PRE) vertical drift, estimated using the time rate of change of F-layer true heights in $5-8 \mathrm{MHz}$ frequency range of digisonde, is found to be about $33 \mathrm{~ms}^{-1}$ at 21:40 UT and bottomside scale height is found to be about $20 \mathrm{~km}$. In the radar Range-time-intensity (RTI) map, the plume (bubble) first appears at 22:10 UT i.e. $30 \mathrm{~min}$ after the pre-reversal peak. The true height variation plot from digisonde does show some degree of wave modulation during
19:00-20:30 UT, an hour and half earlier than the appearance of bubble. However, the modulation does not present any downward phase propagation indicating thereby that the GW activity was rather weak. On the next night 24-25 October, the estimated peak pre-reversal drift and bottomside scale height from digisonde are found to be $18 \mathrm{~ms}^{-1}$ at 21:00 UT and $25 \mathrm{~km}$, respectively, indicating less favorable ionospheric conditions for the excitation of CII on this night as compared to previous night. In the RTI map, the plume first appears at 22:15 UT, i.e. $75 \mathrm{~min}$ later than pre-reversal peak. A periodic modulation and downward phase propagation in $\mathrm{F}$ layer height variation plot prior to bubble appearance is very prominent during 19:00-20:00 LT indicating the presence of strong GW activity as compared to previous night.

Abdu et al. (2009) have further examined these observations theoretically using linear analysis of CII. The linear analysis solves following governing equation for zonal polarization electric field:

$\frac{\partial \delta E_{x}}{\partial t}-\gamma_{R} \delta E_{x}=s_{x}$

where

$\gamma_{R}=\left(-\frac{E_{o x}}{B_{o}}-W_{o y}+\frac{g}{v_{i}}\right) \frac{1}{l_{o}} ;$

$s_{x}=\frac{B_{o} U_{o x}}{l_{o}}\left(\frac{\delta W_{y}}{\kappa_{i}}-\delta W_{x}\right) ; \frac{1}{l_{o}}=\frac{d \log n_{o}}{d y}$

and $E_{o x}, W_{o y}$ and $U_{o x}$ are ambient zonal electric field, vertical neutral wind and zonal ion velocity. $\delta W$ is the perturbed wind associated with GW. This equation is solved for different background conditions and GW amplitudes to infer the influence of GW seeding in the growth of CII.

The observations and linear results presented in a companion paper (Abdu et al., 2009) can be summarized as follows: (1) For the excitation of CII, ambient ionospheric conditions are more favorable on 23-24 October than on 24-25 October; (2) GW activity is stronger on the night of 24-25 October 2005 than on 23-24 October 2005; (3) Linear analysis of CII on 23-24 October reveals an exponential growth of polarization electric field within an hour after seeding; (4) If favorable ambient conditions on this night is replaced with the less-favorable conditions corresponding to 24-25 October, the polarization field does not grow exponentially in next 
two hours after seeding; (5) Polarization field again grew exponentially within an hour under less favorable conditions on 24-25 October if GW activity, which is kept low corresponding to 23-24 October, was raised three times, corresponding to 24-25 October.

Another event consisted of two nights 2-3 and 5-6 October. On 2-3 October, the ionospheric vertical drift reached a peak of $18 \mathrm{~ms}^{-1}$ at 21:20 UT which is followed by a smooth decrease to zero at 22:10 UT. The vertical drift again increased (post-reversal) and attained maximum of $16 \mathrm{~ms}^{-1}$ at 22:30 UT. The bubble in the RTI first appeared at 22:50 UT during post-reversal after this second peak. The true height variation plot presented weak modulation and weak tendency of downward phase propagation which is an indication of weak/marginal GW activity on this night. On 5-6 October, the vertical drift attained maximum of $14 \mathrm{~ms}^{-1}$ at 21:20 UT followed by a downward motion in next $30 \mathrm{~min}$. The ionosphere again moves upward and attained maximum of $12 \mathrm{~ms}^{-1}$ within next one hour. The bubble is seen during this post-reversal phase at 23:40 UT. The downward phase propagation prior to the appearance of bubble is prominent during 19:00-21:00 LT indicating the strong GW activity on this night compared to other night. In summary, it can be said that (6) On 2-3 and 5-6 October 2005, ambient ionospheric conditions are not very different though they were a little less favorable for spread F on 5-6 October; (7) On both nights, the ionosphere undergoes pre-reversal as well as postreversal phase, (8) On, both nights, bubbles are seen during a second phase of up-lift of ionosphere i.e. during post-reversal phase, (9) On both nights, downward GW phase propagation was noticed in the filtered $\mathrm{hF}$ plot from digisonde (Abdu et al., 2009). However, on 2-3 October 2005, the GW activity was weaker than on 5-6 October 2005, (10) Linear growth analysis revealed that polarization field grows exponentially on 2-3 October, (11) It further show weaker growth for a case where ambient conditions correspond to 5-6 October and GW amplitude correspond to 2-3 October, (12) It again revealed exponential growth on night 5-6 October when GW amplitude is raised three times than its amplitude on 2-3 October.

Linear analysis presented in a companion paper (Abdu et al., 2009) only ensures initial growth of CII without taking into account temporal variations of ambient parameters such as vertical drift and rapid varying density owing to the instability itself. These variations may or may not allow exponential growth to continue depending on the nature of variations. This aspect can be examined only with a nonlinear simulation of CII. The two sets of events described above offer ideal conditions to study the effects of GW seeding under varying ionospheric conditions and may be helpful in bringing out additional features concerning seeding mechanism. Hence, we present here several nonlinear simulations of CII for different background conditions and $\mathrm{GW}$ amplitudes that correspond as closely as possible to those observed on 23-24 October/24-25 October and 2-3 October/5-6 October 2005.
The model employed for our study is described in Sect. 3. Section 4 provides a discussion of the model results and their implications for GW seeding of ESF and equatorial plasma bubbles, and our conclusions are presented in Sect. 5.

\section{Model equations and algorithms}

We adopt the following set of equations to study the collisional-interchange instabilities in the equatorial F-region (Kherani et al., 2004):

$$
\begin{aligned}
& \frac{\partial n}{\partial t}+\nabla .\left(n \boldsymbol{u}_{e}\right)=-\beta n-\alpha n^{2} \\
& \nabla . \boldsymbol{J}=e \boldsymbol{\nabla} \cdot\left[n\left(\boldsymbol{u}_{i}-\boldsymbol{u}_{e}\right)\right]=0 . \\
& \boldsymbol{u}_{i, e}=\frac{\kappa_{i, e}}{1+\kappa_{i, e}^{2}} \boldsymbol{v}_{i, e} \times \hat{b}+\frac{1}{1+\kappa_{i, e}^{2}} \boldsymbol{v}_{i, e}
\end{aligned}
$$

where

$v_{i, e}=\frac{-c_{s i, e}^{2}}{v_{i, e n}} \nabla \log n+\frac{\boldsymbol{g}}{v_{i, e n}}+b_{i, e} \boldsymbol{E}+\boldsymbol{W}$

The subscripts " $e$ " and " $i$ " refer to the electrons and ions, respectively. Equations (1) and (2) are the electron continuity and divergence free current $(\boldsymbol{J})$ equations respectively, while $\boldsymbol{u}_{i, e}$ in Eq. (3) are the ion and electron's steady state velocities. The plasma is assumed to be charge-neutral $\left(n_{e}=n_{i}=n\right)$, and it is ensured by Eq. (2). The terms in the right-hand side of Eq. (1) correspond to the chemical loss of electrons by charge exchange process $(\beta)$ and dissociative recombination process $(\alpha)$ respectively. The notations $\kappa_{i, e}$ represent the ratios of the gyro frequencies, $\Omega_{i, e}$, to collision frequencies, $v_{i, e n}$ of the corresponding species, $b_{i, e}=e / m_{i, e} v_{i, e n}$ represent the corresponding mobilities, $\boldsymbol{E}$ is the electric field in the neutral wind $(\boldsymbol{W})$ frame, $\hat{b}$ is the unit vector in the magnetic field, $\boldsymbol{B}$, direction and $c_{s i, e}$ are the ions and electrons thermal velocities respectively. For further analysis, the thermal effects i.e., diffusion effects are neglected restricting our investigation to be applicable only for large scales. The diffusion effects are important to study the saturated turbulent state of the instability. However, in the present investigation, the nonlinear evolution of the CII rather than the saturated turbulent state is investigated. The chemical lossproduction effects are retained in the present study via effective recombination rate $R_{e}$ defined below. Equations (13 ) are solved at magnetic equator in the Cartesian coordinate system where $\hat{x}, \hat{y}$ and $\hat{z}$ correspond to the westward, upward and Earth's magnetic field directions. The dynamics parallel to the magnetic field is ignored and local values of ionospheric parameters, instead of field-line-integrated, are used. This is a limitation of the present simulation which will exclude any study concerning the effects of off-equatorial dynamics on the evolution of equatorial plasma bubble. Assuming the electrostatic perturbation $(\delta E=-\nabla \Phi)$ of electric 
(a) Perturbed GW wind, $\delta W_{o}$

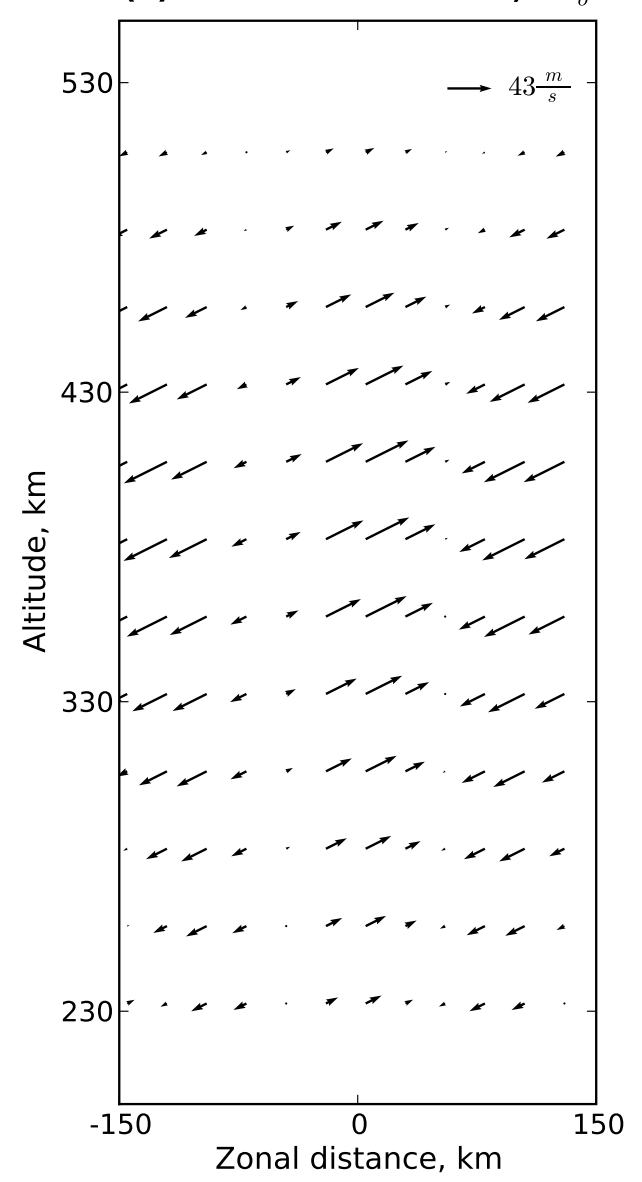

(b) Perturbed ion velocity, $\delta \vec{u}_{i}$

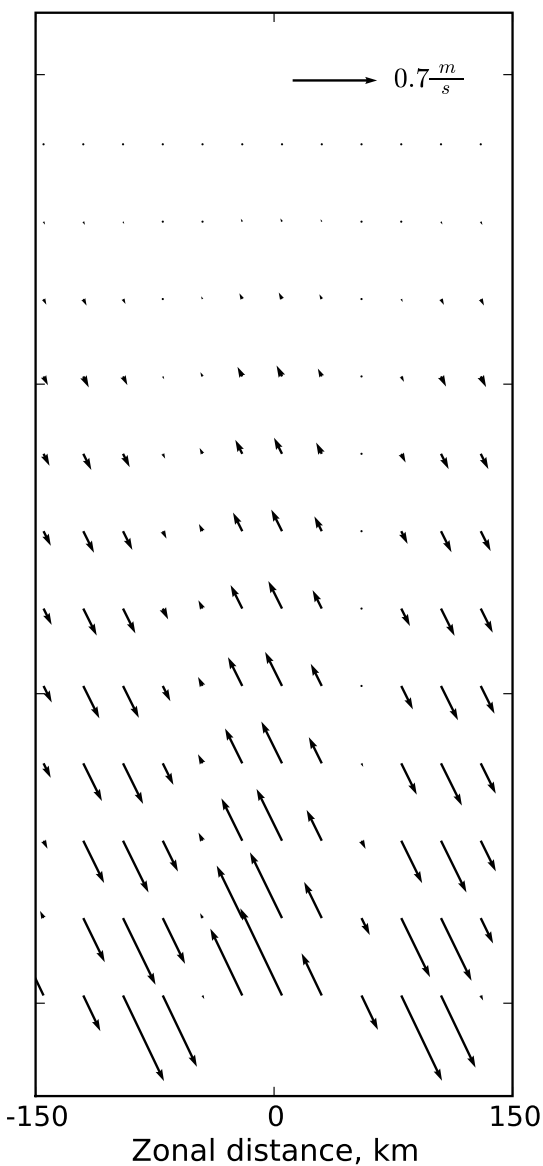

Fig. 1. (a) The distribution of amplitude $\delta W_{o}$ of GW (b) and the perturbed ion velocity $\delta u_{i}$ in the F-region at $t=0$.

field $E=E_{o}+\delta E=E_{o}-\nabla \Phi$, the divergence-free current density Eq. (2) is reduced to the following equation for the perturbed potential $(\Phi)$ in the F-region (Kherani et al., 2004):

$\nabla^{2} \Phi+\frac{\partial \log \left(\kappa_{i} n\right)}{\partial x} \frac{\partial \Phi}{\partial x}+\frac{\partial \log \left(\kappa_{i} n\right)}{\partial y} \frac{\partial \Phi}{\partial y}=s$

and ion continuity Eq. (1) can be written as follows:

$\frac{\partial n}{\partial t}+\boldsymbol{F}(n, \Phi) \cdot \nabla \log n=R_{e} n$

where

$R_{e}=\left(\frac{1}{\beta}+\frac{1}{\alpha n(t)}\right)^{-1}$

is the effective recombination rate and

$\boldsymbol{F}=n\left(\boldsymbol{u}_{o}+\delta \boldsymbol{u}\right) ; \quad \delta \boldsymbol{u}=\frac{1}{\kappa_{i}}\left(\delta \boldsymbol{W}-\kappa_{i} \frac{\nabla \Phi}{B_{o}}\right) \times \hat{b}_{o}$

is the particle flux. Here $u_{o}$ and $\delta u$ are steady-state ambient and pertubed ion velocity respectively. In Eq. (4), $s$ is given by following expression:

$s=-B_{o}\left(\frac{g}{v_{i n}}-\frac{E_{x o}}{B}-W_{y}\right) \frac{\partial \log n}{\partial x}$ or

$s=-B_{o}\left(\gamma_{R}+\delta \gamma\right) l_{o} \frac{\partial \log \delta n}{\partial x}$

where

$$
\begin{aligned}
\gamma_{R} & =\left(\frac{g}{v_{i n}}-\frac{E_{o x}}{B_{o}}-W_{o y}\right) \frac{1}{l_{o}} ; \\
\delta \gamma & =-\frac{\delta W_{y}}{l_{o}} \\
\frac{1}{l_{o}} & =\frac{d \log n_{o}}{d y}
\end{aligned}
$$

Equations (4-5) form a coupled closed system of equations for CII in the F-region. In the expression of $s, \gamma_{R}$ can be recognized as the growth rate of CII. $W_{o}$ appearing in $\gamma_{R}$ represents the background neutral wind and its effects are ignored in present study. The fluctuating perturbed wind $\delta W$ appearing in $\boldsymbol{F}$ and $s$ is associated with the gravity waves. It is noted that this wind has two-fold effects in the F-region of the ionosphere: it modifies the ion velocity (Eq. 3) by introducing perturbation $\delta \boldsymbol{u}$ and redistributes the ionospheric 
(a) Ambient density, $n_{0}$

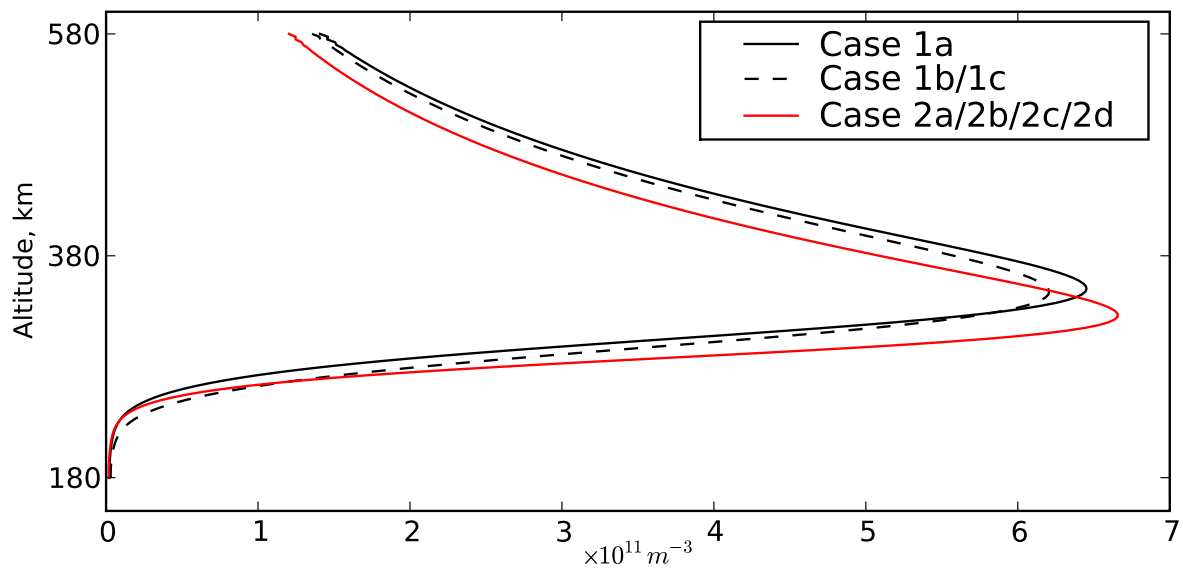

(b) Ambient velocity

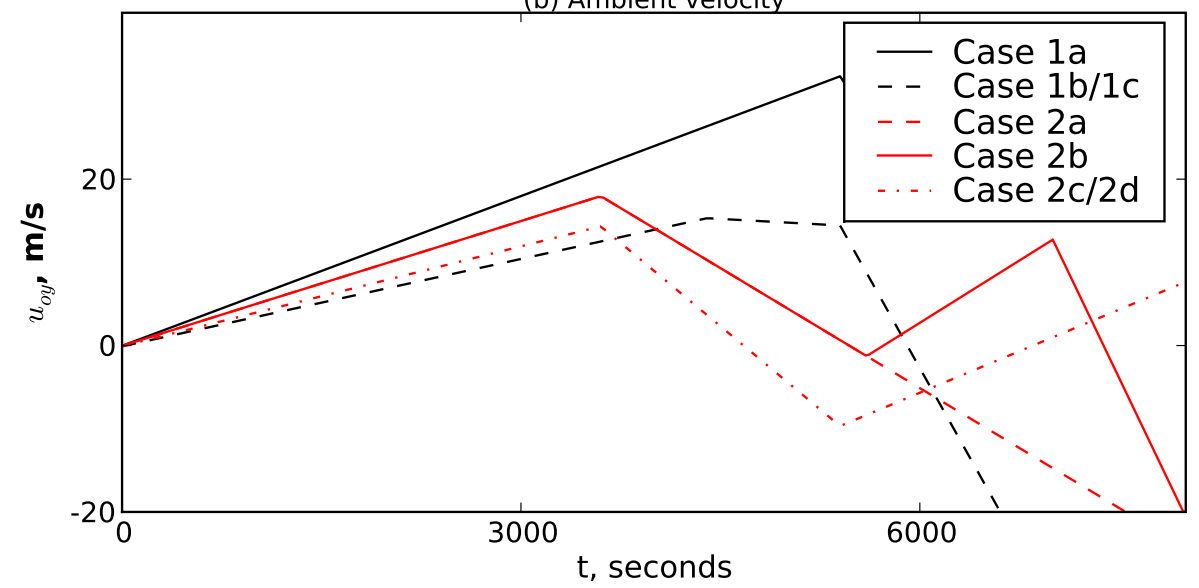

Fig. 2. (a) An altitude profile of initial ionospheric density and (b) temporal variation of ambient vertical velocity chosen for different cases under event 1 and 2. Case 1.a, 1.c, 2.b, 2.d represent ambient ionospheric conditions and GW amplitude as close as possible to the observed spread F nights 23-24, 24-25, 2-3 and 5-6 October 2005, respectively.

density according to the continuity equation (Eq. 5) and it modifies the growth of the instability $\gamma_{R}$ by introducing perturbation $\delta \gamma$ in $s$ which appears in Poisson equation (Eq. 4).

With a chosen seeding perturbation of amplitude $\delta W$ associated with GW and the ambient ionospheric conditions (that are described below and in Figs. 1-2), the continuity Eq. (5) is solved using the Crank-Nicholson implicit scheme at first (Kherani et al., 2004). The potential Eq. (4) is then solved using the Successive-Over-Relaxation algorithm with the perturbed density. This solution is again substituted into Eq. (5) to obtain a time-evolved density and this loop continues till maximum upward velocity of ion becomes $1000 \mathrm{~ms}^{-1}$. The simulation plane consists of $\mathrm{x}$ and $\mathrm{y}$ boundaries in zonal and vertical directions respectively. The $\mathrm{x}$-boundaries are located at $\pm 150 \mathrm{~km}$ with $2.5 \mathrm{~km}$ grid resolution and periodic boundary conditions are imposed on these boundaries. The y-boundaries are located at $180 \mathrm{~km}$ and $580 \mathrm{~km}$ with $2.5 \mathrm{~km}$ grid resolution. The transmittive boundary condition on $n$ and the Neumann boundary condition on $\Phi$ are imposed on these boundaries. These boundary conditions are the same as those chosen by Sekar et al. (1994) and sufficient to ensure the vanishing current density across the lower boundary provided that the ambient ionosphere is invariant at the boundary.

To infer the nature of $\delta \boldsymbol{W}$ of the GW in the present study, we employ a GW vertical propagation model that describes the propagation of a GW from troposphere to thermosphere with specified characteristics at a tropospheric altitude (Kherani et al., 2009). The model provides vertical velocities associated with a GW in $10-600 \mathrm{~km}$ altitude range with a given initial velocity fluctuation at $10 \mathrm{~km}$ altitude. The $\mathrm{GW}$ horizontal velocities are obtained from the continuity equation (Eq. 10 of Fritts et al., 2008b). In the present study, the initial GW vertical velocity amplitude is assumed to be $0.5 \mathrm{~cm} \mathrm{~s}^{-1}$ at $10 \mathrm{~km}$ height. This value is chosen so that wind fluctuation associated with $\mathrm{GW}$ at $80 \mathrm{~km}$ altitude becomes of order of $1 \mathrm{~m} / \mathrm{s}$ which is reported from observations (Fritts et al., 2008b). The vertical and horizontal wavelengths $\left(\lambda_{y, x}\right)$ 
Table 2. Numerical simulation under different events.

\begin{tabular}{lll}
\hline & Ionospheric condition & GW activity \\
\hline Case 1: & & \\
1.a & $23-24$ Oct & $\delta w_{x, y}=40,20 \mathrm{~ms}^{-1}$ \\
1.aa & $23-24$ Oct & $\delta w_{x, y}=20,10 \mathrm{~ms}^{-1}$ \\
$1 . \mathrm{ab}$ & $23-24$ Oct & $\delta w_{x, y}=4,2 \mathrm{~ms}^{-1}$ \\
$1 . \mathrm{b}$ & $24-25$ Oct & $\delta w_{x, y}=40,20 \mathrm{~ms}^{-1}$ \\
$1 . \mathrm{c}$ & $24-25$ Oct & $\delta w_{x, y}=100,50 \mathrm{~ms}^{-1}$ \\
\hline Case 2: & & \\
2.a & $2-3$ Oct (without post-reversal) & $\delta w_{x, y}=40,20 \mathrm{~ms}^{-1}$ \\
2.b & $2-3$ Oct & $\delta w_{x, y}=40,20 \mathrm{~ms}^{-1}$ \\
2.c & 5-6 Oct & $\delta w_{x, y}=40,20 \mathrm{~ms}^{-1}$ \\
2.d & $5-6$ Oct & $\delta w_{x, y}=120,60 \mathrm{~ms}^{-1}$ \\
\hline
\end{tabular}

are chosen to be two and four times larger than the scale height at each altitude. Fixing these wavelengths, the frequency $(\omega)$ of the GW is obtained from the dispersion relation $\omega^{2} \lambda_{x}^{2}=\left(N^{2}-\omega^{2}\right) \lambda_{y}^{2}$ where $N$ is the Brunt-Vaisala frequency. This is one among few options by which the GW's parameters can be selected. An alternative approach is to fix the $\lambda_{y}$ and $\omega$ and calculate the $\lambda_{x}$ from above dispersion relation (Abdu et al., 2009). The wave frequency estimated using the first approach is found to be in the range $10-60 \mathrm{~min}$, depending on the altitude. The wave resides in the equatorial plane $(x-y)$ perpendicular to magnetic field and propagates obliquely at an elevation angle $84^{\circ}$. It means that the wind fluctuations associated with such GWs make an angle of $6^{\circ}$ from horizontal. The fluctuating wind $\delta \boldsymbol{W}_{o}$ associated with the GW is shown in Fig. 1a. It can be seen that $\delta \boldsymbol{W}_{o}$ maximizes in the F-region and it is dominantly horizontal having horizontal wavelength in the range of $200-300 \mathrm{~km}$ and vertical wavelength in the range of $100-150 \mathrm{~km}$. The maximum horizontal and vertical winds associated with GW are of order of $40 \mathrm{~ms}^{-1}$ and $10 \mathrm{~ms}^{-1}$, respectively. This GW, introduces a perturbation $\delta \boldsymbol{u}$ in the ion velocity $\boldsymbol{u}_{o}$ according to Eq. (6). This perturbed velocity is computed using $\delta W$ terms in Eq. (6) and it is plotted in Fig. 1b. It is noted that $\delta u$ is dominantly vertical with a magnitude of $0.5-1 \mathrm{~ms}^{-1}$ in the bottomside F-region.

A SpreadFEx companion paper by Fritts and Vadas (2008) employed the ray tracing methods of Vadas and Fritts (2004) to assess GW Doppler shifting by thermospheric winds, preferential penetration and dissipation, and selection of those GW periods and spatial scales achieving the highest altitudes for various thermospheric winds and solar conditions and arbitrary GW sources in the lower atmosphere. They suggested that those GWs achieving the largest amplitudes will necessarily play the major roles in ionospheric plasma processes and that such GWs typically have relatively high intrinsic frequencies (the observed and the intrinsic periods being $\sim 10$ to $60 \mathrm{~min}$ and $\sim 10$ to $30 \mathrm{~min}$, respectively) and large horizontal and vertical scales (dominant horizontal and vertical wavelengths being $\sim 150$ to $500 \mathrm{~km}$ and $\sim 150$ to $300 \mathrm{~km}$, respectively, for solar conditions representative of SpreadFEx condition). These theoretical estimates appear to be in good agreement with the typical GW periods observed during the SpreadFEx campaign that were found to be in the $\sim 15-60$ min range near $300 \mathrm{~km}$ (Abdu et al., 2009; Fritts et al., 2009). The longer wavelength GWs that are expected to reach F-region altitudes, are also expected to achieve horizontal velocities in the range of $10-100 \mathrm{~ms}^{-1}$ or higher, depending on propagation conditions, specific scales, and proximity to their sources. Corresponding vertical component is typically somewhat smaller, but may also attain similar amplitudes near altitudes at which they approach evanescence. It can be noted that the frequency, wavelengths and amplitudes of GW obtained in present investigation (Fig. 1a-b) fall in the same range as suggested by Fritts and Vadas (2008).

\section{Results and discussion}

Using simulation model described above, two events, which are discussed in Sect. 2 and listed in Table 1, are studied. The First event set consists of 23-24/24-25 October 2005 and second event set consists of 2-3/5-6 October 2005. In next two subsections, we present the numerical results corresponding to these two events respectively.

\subsection{Event 1: 23-24 and 24-25 October 2005}

On 23-24 October/24-25 October, the modulation and downward phase propagation in the true height vs. time plot are seen mainly during 19:00-21:00 UT and pre-reversal enhancement peak occurred almost an hour or more later (Abdu et al., 2009). On this basis, our simulation $(t=0 \mathrm{~s})$ begins at 20:00 LT when GW activities are observed and when the ionosphere upward drift is small. The amplitude of GW on two nights are derived using observed time variation of bottomside F-layer height $(h F)$. The time rate of change of $h F$ at given time represents perturbed upward ion velocity $\delta u$. For one hour periodic modulation observed during 19:0021:00 UT, this time rate is estimated on each night and the corresponding wind perturbation $\delta W_{x} \approx \kappa_{i} \delta u_{y}$ is obtained using Eq. (6). It is found that estimated $\delta u$ is of the order of $1 \mathrm{~ms}^{-1}$ and $2.5 \mathrm{~ms}^{-1}$ on 23-24 October and 24-25 October, respectively. On this basis, we have chosen $\delta \boldsymbol{W}=\delta \boldsymbol{W}_{o}$ and $\delta \boldsymbol{W}=2.5 \delta W_{o}$ for 23-24 October and 24-25 October, respectively, where $\delta W_{o}$ is shown in Fig. 1a. The initial density profile and temporal profile of $u_{o y}=-E_{o x} / B_{o}$ for these cases are shown in Fig. 2a and b. These profiles are deduced from the digisonde observations presented in Abdu et al. (2009). On the basis of ambient ionospheric conditions and GW activity, we consider following cases within the Event 1 (they are also listed in Table 2):

Case 1.a: Ambient condition and GW amplitude corresponding to 23-24 October, i.e. $\delta \boldsymbol{W}=\delta \boldsymbol{W}_{o}$; 
(a) Case 1a
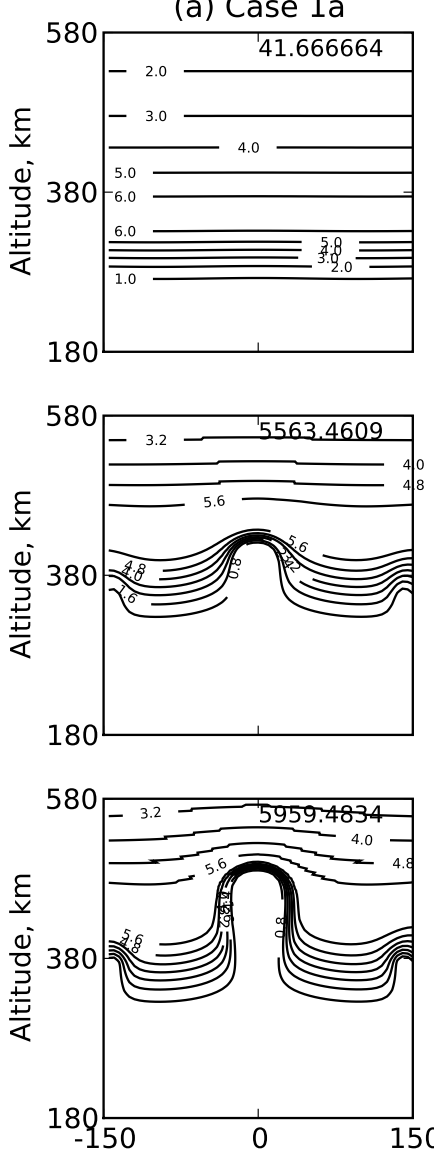

(b) Case $1 \mathrm{~b}$
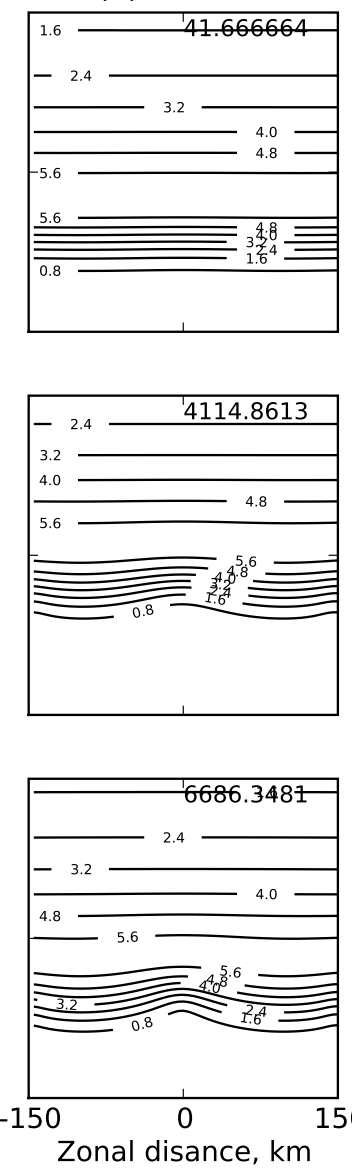

(c) Case 1c
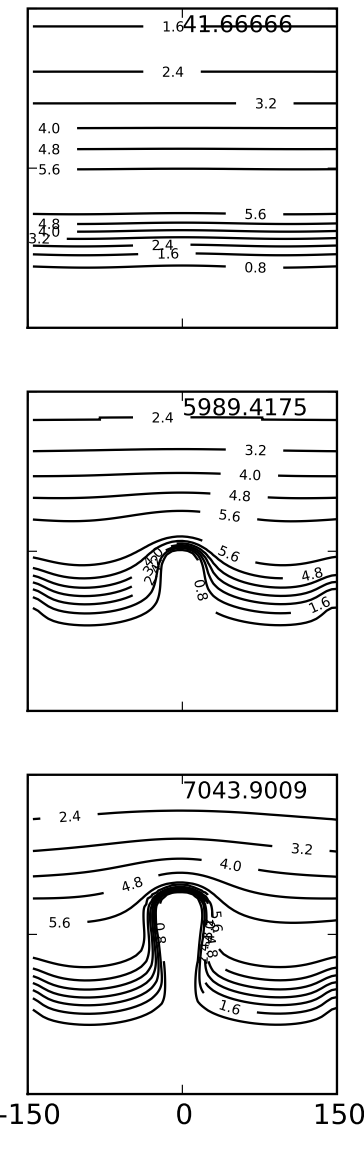

Fig. 3. Event 1: The evolved iso-density contours in the simulation plane for (a) Case 1.a (b) Case 1.b and (c) Case 1.c.

Case 1.b: Ambient condition corresponding to 24-25 Oct, and GW amplitude corresponding to 23-24 October, i.e. $\delta \boldsymbol{W}=\delta \boldsymbol{W}_{o}$;

Case 1.c: Ambient condition and GW amplitude corresponding to 24-25 October, i.e. $\delta \boldsymbol{W}=2.5 \delta \boldsymbol{W}_{o}$;

Case 1.aa and 1.ab: Ambient conditions corresponding to 23-24 October and GW amplitude is lowered by factor 0.5 and 0.2 from its amplitude on 23-24 October.

The results corresponding to the different cases under event 1 are shown in Figs. 3-4. In Fig. 3, the iso-density contours (IDCs) are shown for Case 1.a (left-panels), Case 1.b (middle-panels) and Case 1.c (right panels) during the evolution of CII in each case. For each case, the evolution of CII at different time (written on the top of each panel) are presented. It is evident that the initial perturbations grow as rapidly rising plasma bubbles in Cases 1 .a and 1.c, but not in Case 1.b, highlighting the importance of enhanced GW activity that gives rise to the plasma bubble in Case 1.c under less favorable ambient conditions than in Case 1.a. This aspect is more clear in Fig. 4 where the maximum upward velocity inside a depletion is plotted for Case 1.a-1.c. It is known from earlier simulation studies that large upward velocity inside a depletion is an indication of nonlinear growth of CII (Sekar et al., 1994). The upward velocity is computed from the solution of Eq. (4) using the following expression:

$\delta u_{y}=-\frac{\delta E_{x}}{B_{o}}=\frac{1}{B_{o}} \frac{\partial \Phi}{\partial x}$

We note in Fig. 4 that for Case 1.a corresponding to 23-24 October, plasma depletion evolves rapidly. In contrast, we see almost no growth for Case 1.b, where the GW amplitude is kept same as Case 1.a, but less favorable ambient conditions observed on 24-25 October are imposed. However, under the same less favorable conditions as Case 1.b, but with enhanced GW activity corresponding to 24-25 October, rapid rising bubble are formed though not as rapid as in Case 1.a. These results indicate that though the ambient ionospheric conditions were less favorable to CII and to the excitation of deep plasma bubbles on 24-25 October, bubbles could, nevertheless, develop owing to the enhanced GW activity. In addition, Case 1.aa and 1.ab in Fig. 4 reveal 


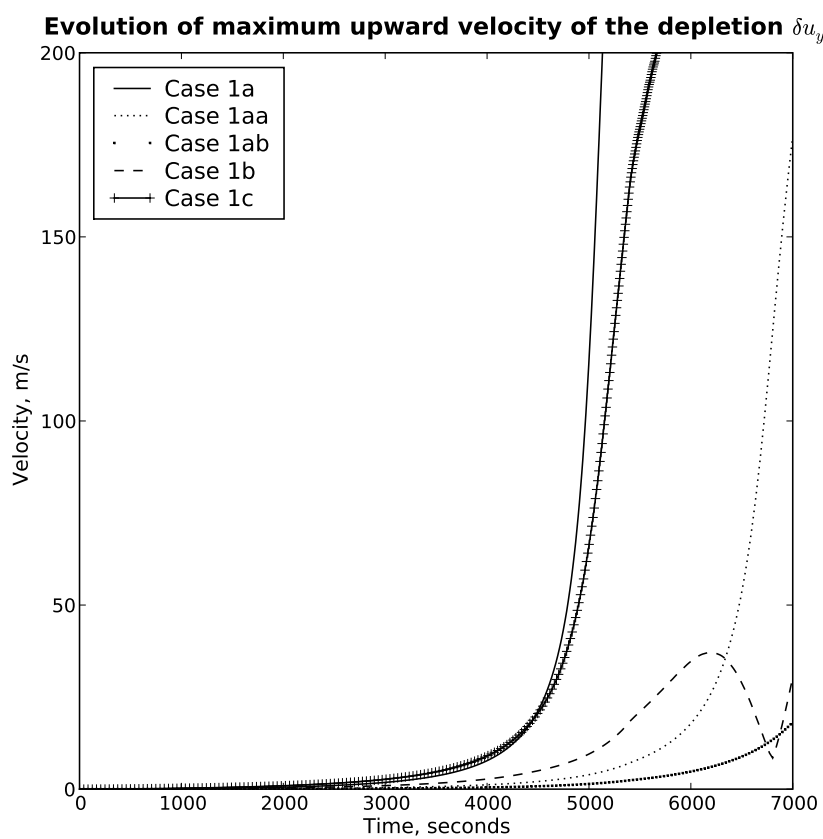

Fig. 4. Event 1: The growth of maximum upward velocity of depletion for Case 1.a-1.c.

marginal growth and no-growth tendency. It means that in spite having highly favorable ambient conditions on 23-24 October, the bubble growth is substantially reduced if the amplitude of seeding GW is lowered. It means that the GW amplitude above certain threshold is needed to seed the instability that can develop into bubble. It is then noted that in spite the large $\delta W$ on 24-25 October than on 23-24 October, CII growth is much faster on 23-24 October 2005. This is attributed to the ambient ionospheric conditions such as vertical drift, density gradient, and F-layer height that dictate the growth of CII, being less favorable on 24-25 October 2005. We note that though GW is needed to seed or initiate the instability, its impact on the growth is dictated by the ambient ionospheric conditions. In essence, all it can be said that the GW with amplitude larger than certain threshold is a suitable seeding for the CII. However, it may or may not cause the growth of CII depending on the ambient ionospheric conditions. The nonlinear features presented in Figs. 3-4 are in accord with the results obtained using linear analysis by Abdu et al. (2009).

Afore-mentioned behavior of CII under varying GW activities can be discussed qualitatively by analyzing the continuity Eq. (5) and source function $s$ in Poisson Eq. (4). The order of density perturbation caused by the $\mathrm{GW}$ wind can be obtained from Eq. (4) as follows:

$\omega \delta n \sim n_{o} \delta u_{y} / \lambda_{x} ;$ or $\quad \frac{\delta n}{n_{o}} \sim \frac{\nu_{i n}}{\Omega_{i}} \frac{\tau \delta w_{x}}{\lambda_{x}}=\frac{v_{i n}}{\Omega_{i}} \frac{\delta w_{x}}{v_{p h}}$ where $\tau=1 / \omega$ and $\lambda_{x}$ are the time-period and wavelength associated with GW and $v_{p h}$ is its phase velocity in x-direction. For $\tau=30 \mathrm{~min}, \lambda_{x}=200 \mathrm{~km}$ and $\delta w_{x}=40 \mathrm{~ms}^{-1}, \delta n / n_{o} \sim 0.5 \%$ i.e. the F-region ionospheric density is perturbed by $0.5-$ $1 \%$ from its ambient value caused by the GW described in Fig. 1a-b. This perturbation appear in source function $s$ which is given by Eq. (7) and can also be written as follows:

$s=B_{o}\left(\gamma_{R}+\delta \gamma\right) \frac{l_{o}}{\delta l_{x}}$

where

$\frac{1}{\delta l_{x}}=\frac{\partial \log n}{\partial x} \sim \frac{1}{\lambda_{x}} \frac{\delta n}{n_{o}} \sim \frac{\tau}{\lambda_{x}^{2}} \frac{v_{i n}}{\Omega_{i}} \delta w_{x}$

Thus $s$ can be written as follows:

$s \sim B_{o}\left(\gamma_{R}-\frac{\delta w_{y}}{l_{o}}\right) \frac{l_{o}}{\lambda_{x}} \frac{\delta w_{x}}{v_{p h}}$

In Case 1.a, $\delta w$ is small but since $\gamma_{R}$ is large, the source function $s$ is sufficiently large for instability to grow. In Case 1.c, $\gamma_{R}$ is smaller (almost by factor 2) than its value in Case 1.a. However, $\delta w$ is larger (by factor 2) than its value in Case 1.c owing to enhanced GW activity. Thus the value of " $s$ " may still remain sufficiently large for the growth of bubble for Case 1.c. For Case 1.b, both $\gamma_{R}$ and $\delta w$ are small implying small $s$ and insignificant growth of CII.

In their numerical simulation of CII with GW as a seeding perturbation, Huang and Kelley (1996) have found that though the plasma bubbles may be produced in the all situations, the production of the plasma bubbles initiated by GWs takes a time much shorter than that resulting from two-dimensional initial density perturbations. It is discussed above that the effects of GW enters to the simulation through variable " $s$ " which is $\gamma_{R}+\delta \gamma$ times $l_{o} / \delta l_{x}$. The GW wind modifies the growth rate $\delta \gamma$ and also gives rise to density perturbation which is associated with $1 / \delta l_{x}$ in "s". In all the cases presented so far, effects of GW in both growth rate and density perturbation are taken. In order to see the effect of density perturbation only, we have chosen the case where $\delta \gamma=0$ in $\gamma$ but $1 / \delta l_{x}$ and other parameters are same as case 1.c. In Fig. 5, the growth of maximum upward velocity of depletion is plotted for this case. It reveals that though CII grows but the growth is prolonged when perturbation is considered only in the density and not in the growth rate. This is in accord with the results found by Huang and Kelley (1996) though the time-difference in Fig. 5 is much smaller than time-difference obtained by them. From Eq. (8), it is evident that the growth rate modification $\delta \gamma$ depends on $\delta W_{y}$ which, in our study, is very small compared to its magnitude used in the study by Huang and Kelley (1996). Thus, the different results in the two studies, in terms of the magnitude, are caused by the different GW parameters used in the simulations. 


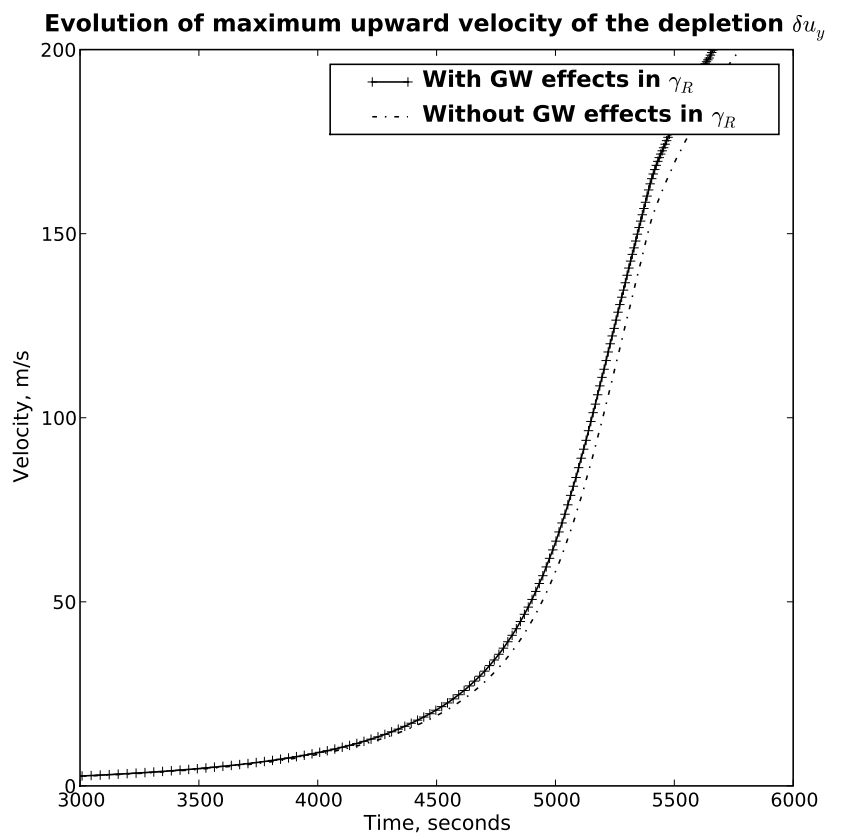

Fig. 5. Event 1: The growth of maximum upward velocity of depletion for case without GW wind $\delta W$ effects on the growth rate $\gamma_{R}$. The ionospheric conditions and GW activities are kept same as in Case 1.c. For comparison, the growth of velocity for Case 1.c is also plotted.

\subsection{Event set 2: 02-03 and 05-06 October 2005}

These are the post-reversal events where deceleration (on 2-3 October) or reversal (5-6 October) of upward drift of ionosphere after pre-reversal is followed by upward drift again. Similar to event 1, the simulation begins at 20:00 LT for event 2, when GW activities are observed and when ionosphere upward drift is small. The GW amplitudes on the two nights are determined from digisonde observations as it was done for event 1 and $\delta W$ is found to be equal to $\delta W_{o}$ and $3 \delta W_{o}$ on 2-3 October and 5-6 October, respectively. To study this event, we have examined the following cases:

Case 2.a: Ambient ionosphere conditions corresponding to 2-3 October but without post-reversal increase in vertical drift and GW amplitude $\delta W=\delta W_{o}$,

Case 2.b: Ambient ionosphere conditions corresponding to 2-3 October including the post-reversal increase in vertical drift and GW amplitude $\delta W=\delta W_{o}$,

Case 2.c: Ambient ionosphere conditions corresponding to 5-6 October and GW amplitude $\delta W=\delta W_{o}$.

Case 2.d: Ambient ionosphere conditions corresponding to 5-6 October and GW amplitude $\delta W=3 \delta W_{o}$.

The Ionospheric number density profile used in all the cases are same and shown in Fig. 2a. The corresponding time variation of ambient vertical drift $u_{o y}$ for these cases are shown in Fig. 2b. In Fig. 6, the growth of maximum velocity of the

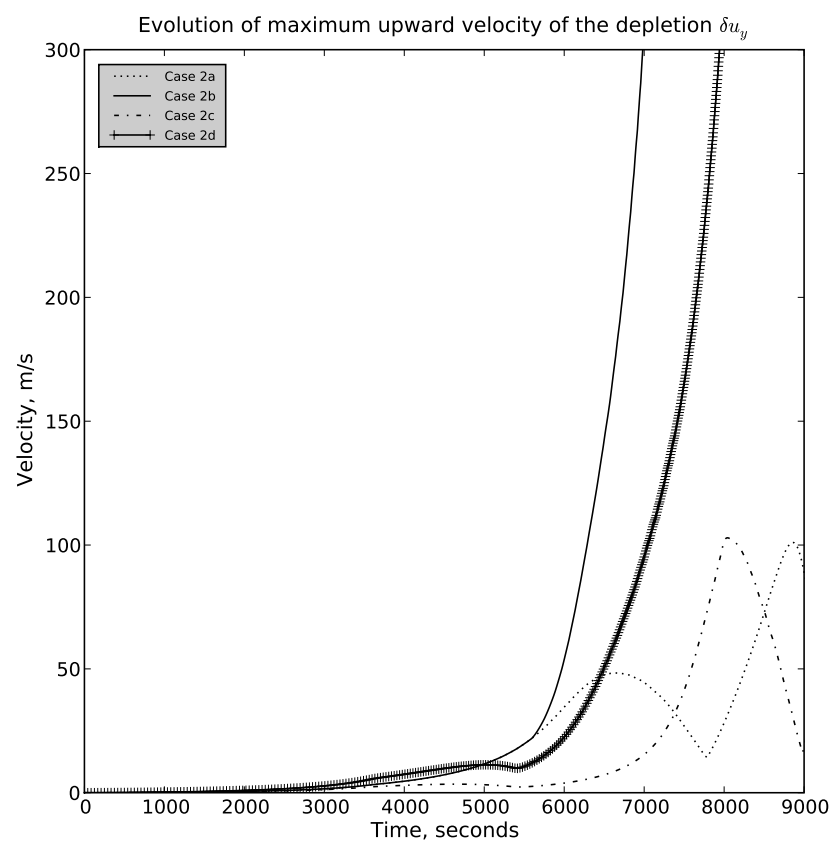

Fig. 6. Event 2: The growth of maximum upward velocity of depletion for Case 2.a-2.d

depletions is plotted for these cases. We note that rapid rising bubbles are formed under Case 2.b and Case 2.d which represent 2-3 October and 5-6 October events, respectively. In Case 2.a, the velocity of depletion remains smaller than $100 \mathrm{~ms}^{-1}$. This implies that a rapid rising bubble will not be formed on 2-3 October if post-reversal upward motion is absent, i.e. when ionosphere continues to move downward after pre-reversal peak. In other words, the post-reversal phase is essential for generation of bubble on this night which is in confirmation with the observation. In contrast to rapid rising bubble for Case 2.d corresponding to 5-6 October, low velocity depletion is noted for Case 2.c. This means that rapid rising bubble would not be formed on 5-6 October if GW amplitude is chosen to be low. In the presence of weak GW, rapid descending ionosphere after pre-reversal on this night will not allow perturbation to grow as bubble. In other words, large GW amplitude is essential on this night to overcome suppressing effects of a rapid descending ionosphere and to gives rise to bubble which is in confirmation with the observation. The nonlinear results corresponding to Case 2.b/2.d are in accordance with the linear results presented in the companion paper by Abdu et al. (2009). For Case 2.c, linear result show growth of CII though much slower than Case 2.d. The present numerical result corresponding to Case 2.c does show depletion moving with $100 \mathrm{~ms}^{-1}$ indicating initial growth of CII. This growth, however, could not continue in the absence of strong GW activity. In Fig. 7, iso-density-contours in the altitude-longitude plane are shown for Case 2.b/2.d. It is evident that on both 
(a) Case $2 b$
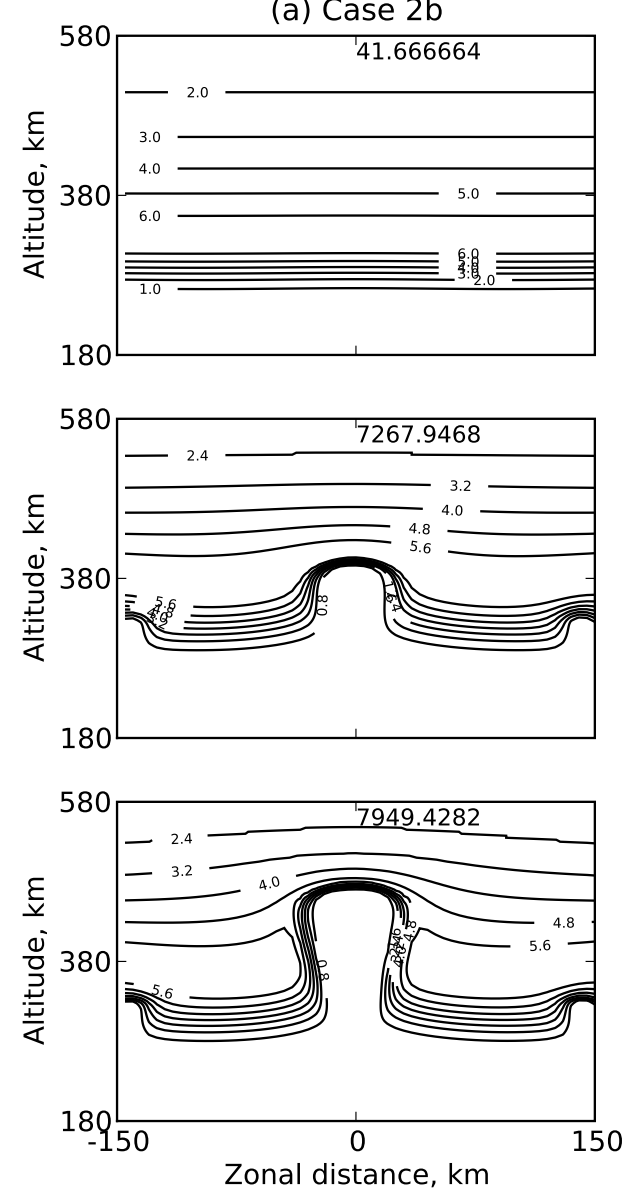

(b) Case $2 d$
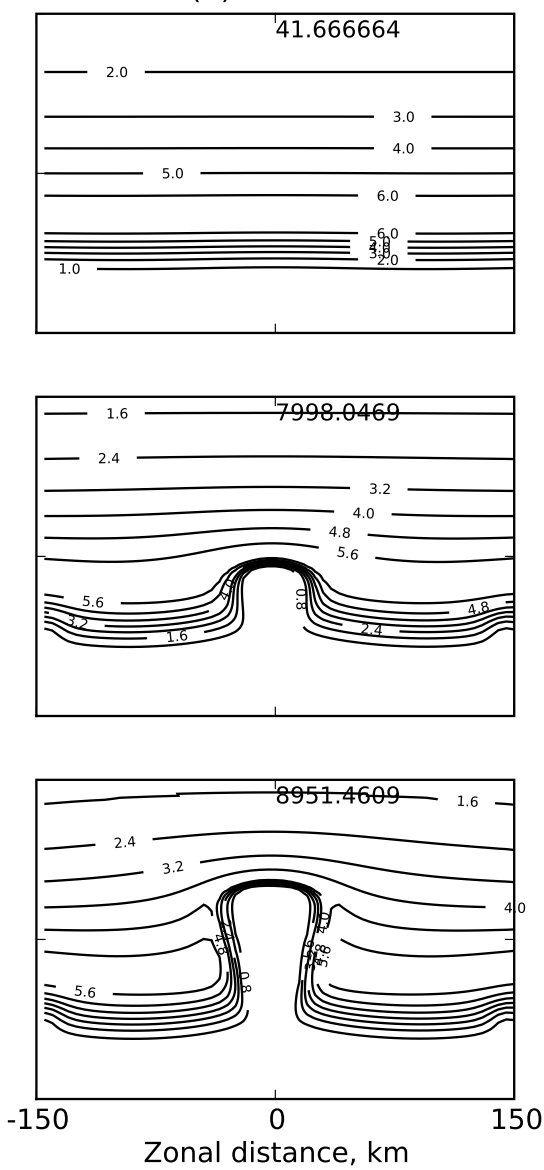

Fig. 7. Event 2: The evolved iso-density contours in the simulation plane for (a) Case 2.b and (b) Case 2.d.

occasions, bubbles evolved much later caused by the second peak in the vertical drift. This kind of behavior of bubble was simulated earlier by Sekar and Kelley (1998) to explain the late emergence of plume over Jicamarca.

\section{Conclusions}

The influences of gravity wave wind perturbations on the evolutions of plasma bubbles are examined for varying ambient ionospheric conditions deduced from observations during the SpreadFEx campaign performed in Brazil from September to November 2005. Nonlinear simulations of collisional interchange instability are performed to accomplish this task. The gravity waves $(\mathrm{GW})$ are assumed to be launched from convective regions in the troposphere. Their upward propagations to thermospheric height are studied using a gravity wave propagation model presented in an earlier work. The gravity wave amplitude in the F-layer is found to be of order of tens of meter per second for an assumed amplitude of $0.5 \mathrm{~cm} \mathrm{~s}^{-1}$ at $10 \mathrm{~km}$ altitude in troposphere. Our results demonstrate that the gravity wave seeding can excite the collisional interchange instability and give rise to plasma bubbles, depending on ambient ionospheric conditions. We have presented a detailed study of two event sets with varying ionospheric conditions and gravity wave amplitudes for these studies.

The first event set corresponds to 23-24 October and 2425 October 2005 for which gravity wave amplitudes are found to be low and high, respectively. The ambient ionospheric conditions are less favorable for the generation of CII on 24-25 October. It is found that a low gravity wave amplitude corresponding to $23-24$ October cannot give rise to a plasma bubble for less favorable ambient conditions representing 24-25 October However, under same less favorable conditions, a bubble can grow if the gravity wave amplitude is increased to a value corresponding to that which characterized the event of 24-25 October. This result is a clear indication of impact of gravity wave seeding on the growth of plasma bubbles.

Another event set corresponds to the nights of 2-3 October and 5-6 October 2005 when the ambient ionospheric 
conditions are not very different, though they are little less favorable on 5-6 October 2005. The GW activity was noted on both days, though it was stronger on 5-6 October. These are the post-reversal events where deceleration or reversal of upward drift of ionosphere after its pre-reversal peak is followed by upward drift again. Our numerical simulation of this event reveals that bubbles are generated during postreversal upward motion of ionosphere on both the nights. The bubbles growth would not have occurred on 5-6 October if the gravity wave amplitude is lowered to its value on 2-3 October, due to the rapid downward motion of ionosphere after the pre-reversal peak.

Summarizing, our nonlinear simulations of collisionalinterchange instabilities for F-layer environments and GW perturbations guided by measurements during the SpreadFEx campaign appear to confirm the suggestions by Fritts et al. (2008b) and the linear plasma instability analysis by Abdu et al. (2009) that GWs can attain sufficiently large amplitudes to significantly enhance CII growth rates and the seed plasma bubbles extending to much higher altitudes. Our sensitivity studies also indicate an important role for gravity waves in the seeding process and suggest that spread $\mathrm{F}$ dynamics can likely not be understood fully without sensitivity to potential gravity wave contributions to the state of the ionosphere at the time of bubble seeding. It is important to mention that more general numerical studies have been performed in the past on the GW seeding mechanism (Huang et al., 1993; Huang and Kelley, 1996). The present study is rather an attempt to provide supportive evidences of gravity wave seeding mechanism on the basis of a few observed events and their numerical simulations.

Acknowledgements. The authors wish to acknowledge the supports from FAPESP through the process 07/00104-0, project 1999/004370 , and CNPq through grants no 502804/2004-1, 500271/2003-8. The SpreadFEx field program and supporting data analyses and modeling were also supported by NASA contracts NNH04CC67C and NAS5-02036 and AFOSR contract FA9550-06-C-0129.

Topical Editor U.-P. Hoppe thanks S. Saito and another anonymous referee for their help in evaluating this paper.

\section{References}

Abdu, M., Alam Kherani, E., Batista, I. S., de Paula, E. R., and Fritts, D. C.: Gravity wave influences on plasma instability growth rates based on observations during the Spread F Experiment (SpreadFEx), Ann. Geophys., in press, 2009.

Fritts, D. C., Abdu, M. A., Batista, B. R., et al.: Overview and summary of spread F experiment (spreadFEx), Ann. Geophys., in review, 2009.

Fritts, D. C. and Vadas, S. L.: Gravity wave penetration into the thermosphere: sensitivity to solar cycle variations and mean winds, Ann. Geophys., 26, 3841-3861, 2008a, http://www.ann-geophys.net/26/3841/2008/.

Fritts, D. C., Vadas, S. L., Riggin, D. M., Abdu, M. A., Batista, I. S., Takahashi, H., Medeiros, A., Kamalabadi, F., Liu, H.-L.,
Fejer, B. G., and Taylor, M. J.: Gravity wave and tidal influences on equatorial spread $\mathrm{F}$ based on observations during the Spread F Experiment (SpreadFEx), Ann. Geophys., 26, 32353252, 2008b, http://www.ann-geophys.net/26/3235/2008/.

Hysell, D. L., Kelley, M. C., Swartz, W. E., and Woodman, R. F.: Seeding and layering of equatorial spread $\mathrm{F}$ by gravity waves, J. Geophys. Res., 95, 17253-17260, 1990.

Huang, C. S., Kelley, M. C., and Hysell, D. L.: Nonlinear RayleighTaylor instabilities, atmospheric gravity waves, and equatorial spread F, J. Geophys. Res., 98, 15631-15642, 1993.

Huang, C. S. and Kelley, M. C.: Nonlinear evolution of equatorial spread F 4. Gravity waves, velocity shear and day-to-day variability, J. Geophys. Res., 101, 24521-24532, 1996.

Kelley, M. C., Larsen, M. F., LaHoz, C., and McClure, J. P.: Gravity wave initiation of equatorial spread F: A case study, J. Geophys. Res., 86, 9087-9100, 1981.

Kelley, M. C., LaBelle, J., and Kudeki, E.: The Condor equatorial spread F campaign: Overview of the results of large scale measurements, J. Geophys. Res., 91, 5487-5503, 1986.

Keskinen, M. J., Ossakow, S. L., and Fejer, B. G.: Threedimensional nonlinear evolution of equatorial ionospheric spread-F bubbles, Geophys. Res. Lett., 30, 1855, doi:10.1029/2003GL017418, 2003.

Kherani, E. A., de Paula, E. R., and Bertoni, F. C. P.: Effects of the fringe field of Rayleigh-Taylor instability in the equatorial E and valley regions, J. Geophys. Res., 109, A12310, doi:10.1029/2003JA010364, 2004.

Kherani E. A., Mascarenhas, M., Sobral, J. H. A., de Paula, E. R., and Bertoni, F. C.: A three dimension simulation model of collisional interchange instability, Space Sci. Rev., 121, 253-269, 2005.

Kherani E. A., Lognonne, P., Nishant Kamath, Crespon, F., and Garcia, R.: Response of the Ionosphere to the seismic trigerred acoustic wave: electron density and electromagnetic fluctuations, Geophys. J. Int., 176, 1-13, 2009.

McClure, J. P., Singh, S., Bamgboye, D. K., Johnson, F. S., and Kil, H.: Occurrence of equatorial F-region irregularities: Evidence for tropospheric seeding, J. Geophys. Res., 103, 29119-29135, 1998.

Ogawa, T., Ostuka, Y., Shiokawa, K., Saito, A., and Nishioka, M.: Ionospheric disturbances over Indonesia and their possible association with atmospheric gravity waves from the troposphere, J. Meteorological Society of Japan, 84A, 327-342, 2006.

Ossakow, S. L., Zalesak, S. T., McDonald, B. E., and Chaturvedi, P. K.: Nonlinear equatorial spread F: Dependence on altitude of the $F$ peak and bottom-side background electron density gradient scale length, J. Geophys. Res., 84, 17-29, 1979.

Raghavarao, R., Sekar, R., and Suhasini, R.: Nonlinear numerical simulation of equatorial spread $\mathrm{F}$ - Effects of winds and electric fields, Adv. Space Res., 12, 227-230, 1992.

Rottger, J.: Equatorial spread F by electric fields and atmospheric gravity waves generated by thunderstorms, J. Atmos. Terr. Phys., 43, 453-462, 1981.

Scannapieco, A. J. and Ossakow, S. L.: Nonlinear equatorial spread F, Geophys. Res. Lett., 3, 451-454, 1976.

Sekar, R., Suhasini, R., and Raghavarao, R.: Effects of vertical winds and electric fields in the nonlinear evolution of equatorial spread F, J. Geophys. Res., 99, 2205-2213, 1994.

Sekar, R. and Kelley, M.: On the combined effects of vertical shear 
and zonal electric field patterns on nonlinear equatorial spread $\mathrm{F}$ evolution, J. Geophys. Res., 103(A9), 20735-20747, 1998.

Singh, S., Johnson, F. S., and Power, R. A.: Gravity wave seeding of equatorial plasma bubbles, J. Geophys. Res., 102, 7399-7410, 1997.

Sultan, P.: Linear theory and modelling of the Rayleigh-Taylor instability leading to the occurrence of equatorial spread F, J. Geophys. Res., 101, 26875-26891, 1996.

Vadas, S. L.: Horizontal and vertical propagation, and dissipation of gravity waves in the thermosphere from lower atmospheric and thermospheric sources, J. Geophys. Res., 112, A06305, doi:10.1029/2006JA011845, 2007.
Vadas, S. L. and Fritts, D. C.: Thermospheric responses to gravity waves arising from mesoscale convective complexes, J. Atmos. Solar Terres. Phys., 66, 781-804, 2004.

Zalesak, S. T.: Fully multidimensional flux-corrected transport algorithms for fluids, J. Comp. Phys., 31, 335-362, 1979.

Zargham, S. and Seyler, C. E.: Collisional Interchange instability: Numerical simulations of intermediate-scale irregularities, J. Geophys. Res., 92, 10073-10080, 1987. 